\title{
EFICIÊNCIA DE SEPARAÇÃO DE HIDROCICLONES PARA A SEPARAÇÃO DE MISTURA ÓLEO-ÁGUA COM ALTO TEOR DE ÓLEO: COMPARAÇÃO ENTRE RESULTADOS SIMULADOS E EXPERIMENTAIS
}

\author{
C. A. O. DE ARAÚJO ${ }^{1 *}$, C. M. SCHEID², T. S. KLEIN³ ${ }^{3}$ J. B. R. LOUREIRO ${ }^{4}$, R. A. \\ $\mathrm{MEDRONHO}^{3}$ \\ ${ }^{1}$ Universidade Federal dos Vales do Jequitinhonha e Mucuri, Instituto de Ciência Engenharia e \\ Tecnologia \\ ${ }^{2}$ Universidade Federal Rural do Rio de Janeiro, Departamento de Engenharia Química \\ ${ }^{3}$ Universidade Federal do Rio de Janeiro, Departamento de Engenharia Química \\ ${ }^{4}$ Universidade Federal do Rio de Janeiro, Departamento de Engenharia Mecânica \\ e-mail: cristianoagenor@ufvjm.edu.br
}

\begin{abstract}
RESUMO
A utilização de hidrociclones para promover separações baseadas no campo centrífugo iniciou-se há mais de um século. A primeira patente de hidrociclone foi depositada no final do século XIX e sua utilização era para misturas sólido-líquido. Sua utilização para separar misturas líquido-líquido, especialmente óleo-água, foi iniciada em 1970 com pesquisas lideradas pela universidade de Southampton na Inglaterra. A evolução em sua geometria para a separação água-óleo culminou em entradas duplas e duas seções cônicas para aumentar a eficiência de separação. As vantagens em sua utilização são devido ao fato de serem equipamentos compactos, com baixos custos operacionais e que aceleram a separação devido à ação centrífuga. Neste trabalho, empregou-se fluidodinâmica computacional (CFD) e um planejamento fatorial reduzido $\left(2^{7-3}\right)$ para a otimização da geometria de um hidrociclone visando à separação de dispersões de elevados teores de óleo (40\%) em água. O hidrociclone ótimo resultante deste planejamento foi construído e testado experimentalmente. Valores de eficiência de separação $(\mathrm{ET})$ e razão de fluido $\left(\mathrm{R}_{\mathrm{F}}\right)$ foram obtidos experimentalmente e comparados com os valores simulados (ANSYS Fluent 12.1). Experimentos também foram realizados para verificar a influência da razão de fluido total $\left(\mathrm{R}_{\mathrm{FT}}\right)$ sobre a eficiência de separação.
\end{abstract}

\section{INTRODUÇÃO}

Os hidrociclones são equipamentos compactos empregados nas separações sólido/líquido, sólido/sólido, líquido/líquido e gás/líquido. Sua geometria é basicamente constituída por uma parte cilíndrica, que define o diâmetro do hidrociclone, acoplada a uma parte cônica, como mostra a Figura 1.
Os trabalhos disponíveis na literatura empregando hidrociclones para a separação óleo-água tratam apenas de correntes com baixas composições da fase óleo. Suas aplicações se restringem basicamente ao tratamento de águas com quantidade residual de óleo, e que geralmente não ultrapassam o valor de $1 \%(\mathrm{p} / \mathrm{p}$ ou v/v). Nos trabalhos de Bai et al. (2011), Zhou et al. (2010), Almeida et al. (2009) e Nezhati \& Thew (1987) é 
possível confirmar a utilização de baixas concentrações de óleo.

Figura 1 - Desenho esquemático de um hidrociclone.

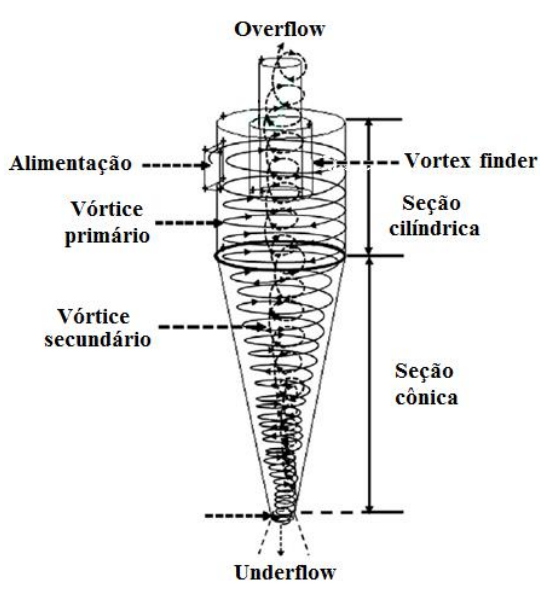

Mesmo com a escassez de trabalhos na literatura sobre a separação de misturas de óleo-água com alto teor de óleo envolvendo hidrociclones, há uma demanda industrial para a substituição dos separadores gravitacionais encontrados em plataformas de petróleo. Estes tanques recebem diretamente os fluidos (óleo, água e gás) provenientes dos poços e a separação gravitacional se dá pela diferença de densidade entre os fluidos envolvidos. Este processo apresenta desvantagens como a necessidade de grandes equipamentos para uma separação eficiente, extensos períodos para uma separação completa das fases e a necessidade de aquecer o sistema para melhorar a separação (Hashmi et al., 2004).

A vantagem dos hidrociclones na limpeza da água a partir da mistura óleo-água, em plataformas marítimas de produção de petróleo, reside não só na maior eficiência em relação aos separadores gravitacionais, mas também na menor área de convés e menor carga sobre o mesmo, devido ao fato destes equipamentos serem mais compactos (Moraes, 2006). Como os hidrociclones atendem a estas especificações, eles têm sido alvo de pesquisas para esta finalidade.

\section{MATERIAIS E MÉTODOS}

As dimensões dos hidrociclones neste estudo foram obtidas com base na observação das proporções geométricas, disponíveis na literatura, para a separação óleo-água envolvendo hidrociclones e também com base nos trabalhos anteriores do Laboratório de Fluidodinâmica Computacional (DEQ/UFRJ). Podemos ressaltar os trabalhos de Freitas et al. (2009); Almeida et al. (2009); Alves \& Medronho (2011, 2012); Coelho et al. (2011, 2012); Anjos et al. (2012); Queiroz \& Medronho (2012); Araújo et al. (2012, 2013); Mendonça et al. (2013) e Braga et al. (2013), que também foram simulados numericamente.

A Figura 2 mostra um esquema do hidrociclone ressaltando todas as sete variáveis geométricas deste estudo. O diâmetro da parte cilíndrica (Dc) e o diâmetro compreendido entre as duas seções cônicas (D) foram mantidos constantes, com valores de $70 \mathrm{~mm}$ e $40 \mathrm{~mm}$, respectivamente.

Figura 2 - Esquema do hidrociclone deste estudo para separação óleo-água, mostrando as variáveis do planejamento fatorial.

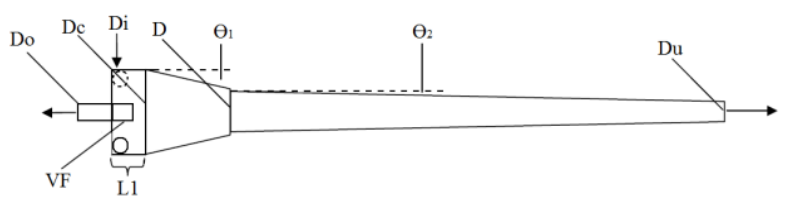

Os intervalos de cada uma das sete variáveis em questão são mostrados na Tabela 1 .

As propriedades físicas da água e do óleo no estudo em questão podem ser vistas na Tabela 2 a seguir. 
Tabela 1- Faixa de cada uma das sete variáveis do hidrociclone estudadas no planejamento fatorial deste trabalho

\begin{tabular}{c}
\hline Intervalo das variáveis \\
\hline $5 \mathrm{~mm} \leq \mathrm{Do} \leq 20 \mathrm{~mm}$ \\
$10 \mathrm{~mm} \leq \mathrm{Du} \leq 25 \mathrm{~mm}$ \\
$4 \mathrm{~mm} \leq \mathrm{VF} \leq 40 \mathrm{~mm}$ \\
$25 \mathrm{~mm} \leq \mathrm{L} 1 \leq 45 \mathrm{~mm}$ \\
$7,5 \mathrm{~mm} \leq \mathrm{Di} \leq 17,5 \mathrm{~mm}$ \\
$2,5^{\circ} \leq \theta_{1} \leq 7,5^{\circ}$ \\
$1^{\circ} \leq \theta_{2} \leq 7,5^{\circ}$ \\
\hline
\end{tabular}

Tabela 2 - Propriedades físicas da água e do óleo

\begin{tabular}{cl}
\hline \multicolumn{2}{c}{ Propriedades físicas } \\
\hline \multirow{2}{*}{ Água } & $\begin{array}{l}\rho=996,5 \mathrm{~kg} / \mathrm{m}^{3} \\
\mu=0,855.10^{-3} \mathrm{~kg} /(\mathrm{m} \mathrm{s})\end{array}$ \\
\hline \multirow{2}{*}{ Óleo } & $\begin{array}{l}\rho=840 \mathrm{~kg} / \mathrm{m}^{3} \\
\mu\end{array}$ \\
& $\mu=13,2.10^{-3} \mathrm{~kg} /(\mathrm{m} \mathrm{s})$ \\
\hline
\end{tabular}

Todas as simulações foram realizadas no Fluent (ANSYS 12.1), em regime permanente. Devido ao escoamento espiralado (forte campo centrífugo) no interior do hidrociclone, o modelo de turbulência utilizado foi o Reynolds Stress Model, proposto por Gibson \& Launder (1978). Quanto à abordagem multifásica, o modelo euleriano foi utilizado para a resolução das equações de conservação. A condição de contorno selecionada para a entrada foi a velocidade de entrada do fluido, velocity inlet, de $4 \mathrm{~m} \mathrm{~s}^{-1}$ para cada uma das duas entradas. Nas duas saídas, over e underflow, foi utilizada a pressão de saída (1 atm), pressure outlet, onde o fluido tem a possibilidade de entrar ou sair do volume de controle de acordo com as condições do escoamento naquela região. Nas paredes do equipamento foi utilizada a condição de aderência, no slip wall. Para todas as simulações, a concentração de óleo (fase dispersa) foi de $40 \%$ (v/v). O diâmetro de gota adotado para o óleo foi de $250 \mu \mathrm{m}$.
Um teste de malha foi conduzido empregando malhas com $100 \mathrm{mil}, 200 \mathrm{mil}$, 400 mil e 600 mil elementos, acompanhandose o perfil de velocidades tangenciais em 4 diferentes alturas do hidrociclone. Com base nestas figuras, foi possível concluir que uma malha de aproximadamente $400 \mathrm{mil}$ elementos era suficiente para estabilizar estes perfis de velocidade. Um teste quanto ao número de iterações mostrou serem necessárias 30.000 iterações para estabilizar a eficiência granulométrica e a razão de fluido, além de garantir o fechamento do balanço de massa.

Para os experimentos com a mistura óleo-água o sistema operou de maneira aberta, isto é, a água e o óleo não foram devolvidos para o tanque de armazenamento, como mostra o esquema da Figura 3. Rotâmetros da marca AppliTech ${ }^{\circledR}$ foram utilizados para medir as vazões de água e de óleo. As vazões volumétricas para o óleo e água foram de 0,51 $\mathrm{m}^{3} \mathrm{~h}^{-1}$ e $0,74 \mathrm{~m}^{3} \mathrm{~h}^{-1}$, respectivamente. O óleo foi bombeado por uma bomba de deslocamento positivo NEMO® BY da marca NETZSCH.

Figura 3: Esquema da unidade experimental construída para as medidas de eficiência de separação da mistura óleo-água.

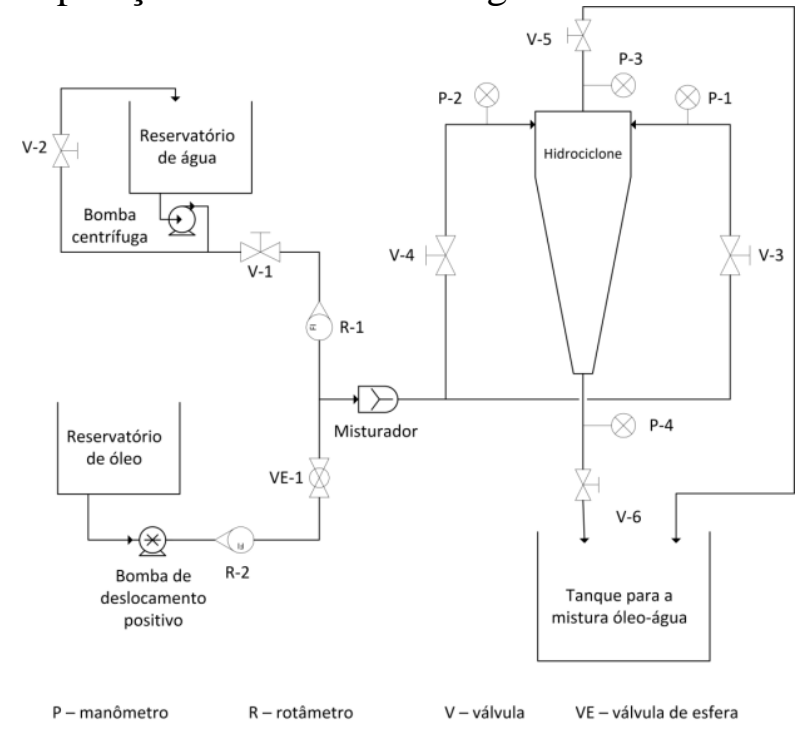




\section{RESULTADOS E DISCUSSÃO}

\subsection{Resultados Simulados: Planejamento Fatorial}

A Tabela 3 mostra os valores das variáveis geométricas para as dezessete simulações. Também são mostrados os valores de eficiência granulométrica reduzida $\left(G^{\prime}\right)$ e de razão de fluido $\left(R_{F}\right)$ obtidos por meio de simulações em regime permanente.
Para todas as simulações do planejamento, o diâmetro das gotas de óleo foi fixado em 250 $\mu \mathrm{m}$.

Os hidrociclones HC 8 e HC 12 foram os hidrociclones que atingiram alto grau de eficiência e valores baixos para razão de fluido. Desses dois hidrociclones, o hidrociclone HC 12 foi alvo de investigação neste trabalho devido o fato de possuir o maior valor de eficiência granulométrica reduzida.

Tabela 3 - Dimensões geométricas e valores de $\mathrm{G}^{\prime}$ e $\mathrm{R}_{\mathrm{f}}$ para o planejamento fatorial reduzido $2^{7-3}$, com partículas de $250 \mu \mathrm{m}$ de diâmetro

\begin{tabular}{cccccccccc}
\hline Simulação & Do (mm) & Du (mm) & $\begin{array}{c}\mathbf{V F} \\
(\mathbf{m m})\end{array}$ & $\begin{array}{c}\mathbf{L 1} \\
(\mathbf{m m})\end{array}$ & $\begin{array}{c}\mathbf{\Theta}_{\mathbf{1}} \\
(\mathbf{r a d})\end{array}$ & $\begin{array}{c}\mathbf{\Theta}_{\mathbf{2}} \\
(\mathbf{r a d})\end{array}$ & $\begin{array}{c}\mathbf{D i} \\
(\mathbf{m m})\end{array}$ & $\begin{array}{c}\mathbf{G}^{\prime} \\
(\boldsymbol{\%})\end{array}$ & $\begin{array}{c}\mathbf{R}_{\mathbf{f}} \\
(\boldsymbol{\%})\end{array}$ \\
\hline 1 & 5 & 10 & 4 & 25 & 0,0872 & 0,0349 & 7,5 & 33,95 & 0,40 \\
2 & 20 & 10 & 4 & 25 & 0,2618 & 0,0349 & 17,5 & 99,97 & 62,20 \\
3 & 5 & 25 & 4 & 25 & 0,2618 & 0,2618 & 7,5 & 0,00 & 0,00 \\
4 & 20 & 25 & 4 & 25 & 0,0872 & 0,2618 & 17,5 & 49,50 & 1,80 \\
5 & 5 & 10 & 40 & 25 & 0,2618 & 0,2618 & 17,5 & 9,42 & 8,50 \\
6 & 20 & 10 & 40 & 25 & 0,0872 & 0,2618 & 7,5 & 57,92 & 94,40 \\
7 & 5 & 25 & 40 & 25 & 0,0872 & 0,0349 & 17,5 & 0,00 & 0,00 \\
8 & 20 & 25 & 40 & 25 & 0,2618 & 0,0349 & 7,5 & 94,87 & 1,00 \\
9 & 5 & 10 & 4 & 45 & 0,0872 & 0,2618 & 17,5 & 41,36 & 0,00 \\
10 & 20 & 10 & 4 & 45 & 0,2618 & 0,2618 & 7,5 & $(-)$ & 100,00 \\
11 & 5 & 25 & 4 & 45 & 0,2618 & 0,0349 & 17,5 & 0,00 & 0,00 \\
12 & 20 & 25 & 4 & 45 & 0,0872 & 0,0349 & 7,5 & 99,70 & 3,40 \\
13 & 5 & 10 & 40 & 45 & 0,2618 & 0,0349 & 7,5 & 16,50 & 4,60 \\
14 & 20 & 10 & 40 & 45 & 0,0872 & 0,0349 & 17,5 & 99,92 & 58,20 \\
15 & 5 & 25 & 40 & 45 & 0,0872 & 0,2618 & 7,5 & 1,67 & 0,40 \\
16 & 20 & 25 & 40 & 45 & 0,2618 & 0,2618 & 17,5 & 32,33 & 10,32 \\
17 (PC) & 12,5 & 17,5 & 22 & 35 & 0,1745 & 0,1483 & 12,5 & 28,93 & 0,19 \\
\hline
\end{tabular}

$\mathrm{HC}$ - hidrociclone

\subsection{Resultados Experimentais com a mistura óleo-água}

O hidrociclone 12 foi construído (Figura 4) e experimentos com concentrações volumétricas de $40 \%$ de óleo em água foram realizados em um sistema aberto para verificar os valores de eficiência total do equipamento. 


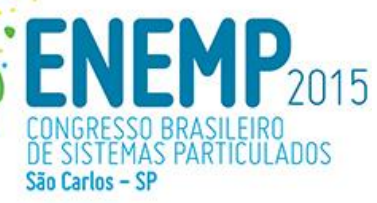

Figura 4: Hidrociclone 12 construído em acrílico para as medidas experimentais.

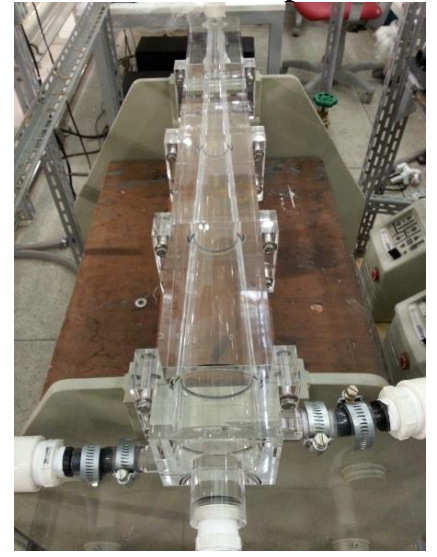

Os valores de vazão de entrada foram idênticos aos das simulações. A vazão para cada entrada foi de $0,635 \mathrm{~m}^{3} \mathrm{~h}^{-1}$. A razão de fluido total foi regulada para $40 \%$ por meio do fechamento da válvula do underflow também de acordo com os dados de simulação. A Figura 5 mostra uma imagem da mistura óleo-água sendo separada dentro do hidrociclone.

Figura 5 - Fração de óleo no interior do hidrociclone 12 .

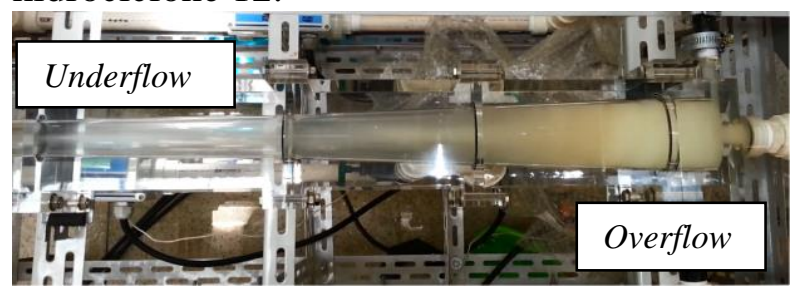

A região esbranquiçada dentro do hidrociclone e próximo ao overflow representa uma zona de alta concentração de óleo. As concentrações de óleo foram medidas nas correntes de entrada e também nas duas correntes de saída. Amostras foram coletadas em uma proveta durante os experimentos, resultando em concentrações baseadas em volume. A Figura 6 mostra um resultado típico (Experimento 8) para as amostras coletadas em um experimento para as correntes de entrada, overflow e underflow, respectivamente.
A queda de pressão empregada neste experimento foi de 0,22 bar. Vale observar que o petróleo produzido normalmente chega à plataforma com pressões superiores a 13 bar $\mathrm{e}$, no tratamento convencional, tem que ter esta pressão reduzida para próximo da pressão atmosférica para ser tratado no separador trifásico gravitacional. Consequentemente, já existe, no fluido a ser tratado, energia suficiente para o tratamento alternativo com hidrociclones.

Figura 6 - Alíquotas da mistura óleo-água coletada na entrada (a), overflow (b) e underflow (c).

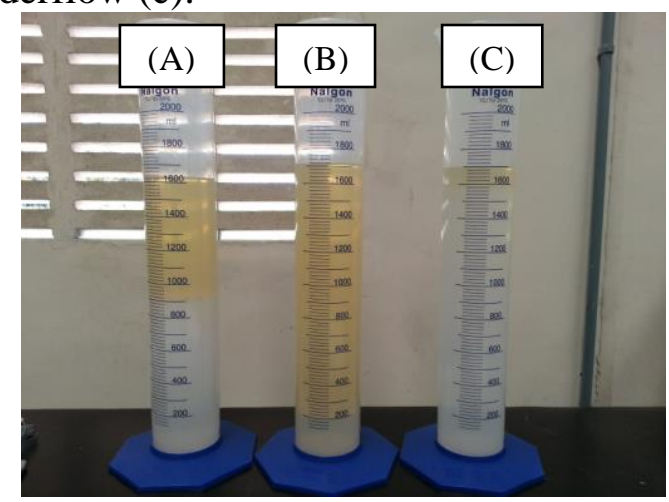

Essas alíquotas mostram de maneira geral os resultados para apenas uma amostra coletada em cada uma das correntes. Mas essas amostras foram coletadas em triplicata e a média desses valores foi obtida. A Tabela 4 mostra os resultados das três amostras e o seu valor médio (Experimento 8), realizado para a mesma condição da simulação.

Tabela 4 - Concentração de óleo (v/v) nas correntes de entrada, overflow e underflow (Experimento 8)

\begin{tabular}{cccc}
\hline Correntes & Entrada & Overflow & Underflow \\
\hline Amostra 1 & $41,50 \%$ & $88,40 \%$ & $7,80 \%$ \\
Amostra 2 & $42,40 \%$ & $88,90 \%$ & $7,10 \%$ \\
Amostra 3 & $44,80 \%$ & $88,80 \%$ & $5,80 \%$ \\
Média & $42,90 \% \pm 1,70$ & $88,70 \% \pm 0,26$ & $6,90 \% \pm 1,01$ \\
\hline
\end{tabular}

Os valores médios apresentados na tabela mostram que a concentração de óleo na corrente de entrada ficou bem próxima do 
valor desejado, que era de $40 \%$. O valor de $88,70 \%$ para a concentração de óleo na corrente do overflow e de apenas $6,90 \%$ na corrente do underflow mostra que o equipamento está separando de maneira adequada, uma vez que as correntes do overflow e underflow estão ricas em óleo e água, respectivamente.

Foram conduzidos três conjuntos de experimentos realizados com a mistura óleoágua. No primeiro, realizaram-se três experimentos idênticos com a mesma vazão de entrada e concentração de óleo das simulações bifásicas, a fim de testar a repetitividade do procedimento experimental (Tabela 5). É possível verificar que na Tabela 5, os experimentos apresentaram repetitividade e valores médios com desvios abaixo de $2 \%$.

Tabela 5 - Eficiência total de separação e razão de fluido experimental realizado em triplicada nas mesmas condições da simulação bifásica

\begin{tabular}{ccccc}
\hline Exp. & 1 & 2 & 3 & Média \\
\hline $\mathrm{Q}_{\mathrm{t}}(\mathrm{L} / \mathrm{min})$ & 21,30 & 20,77 & 20,73 & $20,93 \pm 0,03$ \\
$\mathrm{Q}_{\text {ovf }}(\mathrm{L} / \mathrm{min})$ & 8,93 & 9,11 & 7,48 & $8,50 \pm 1,02$ \\
$\mathrm{Q}_{\text {und }}(\mathrm{L} / \mathrm{min})$ & 12,36 & 11,66 & 13,25 & $12,42 \pm 0,83$ \\
$\mathrm{C}_{\mathrm{v} \text { (alim) }}$ & 0,40 & 0,39 & 0,40 & $0,39 \pm 0,01$ \\
$\mathrm{C}_{\mathrm{v} \text { (ovf) }}$ & 0,89 & 0,87 & 0,90 & $0,88 \pm 0,02$ \\
$\mathrm{C}_{\mathrm{v} \text { (und) }}$ & 0,05 & 0,03 & 0,05 & $0,04 \pm 0,01$ \\
$\mathrm{R}_{\mathrm{FT}}$ & 0,42 & 0,43 & 0,41 & $0,43 \pm 0,02$ \\
$\mathrm{R}_{\mathrm{F}}$ & 0,06 & 0,09 & 0,06 & $0,07 \pm 0,02$ \\
$\mathrm{ET}$ & 0,93 & 0,95 & 0,92 & $0,93 \pm 0,02$ \\
ET $^{\prime}$ & 0,93 & 0,95 & 0,91 & $0,93 \pm 0,02$ \\
\hline
\end{tabular}

No segundo conjunto de experimentos, buscou-se a influência da razão de fluido total sobre a eficiência total de separação do hidrociclone (Tabela 6). A Figura 7 apresenta a variação desta eficiência com a razão de fluido total.
Tabela 6 - Parâmetros medidos nos experimentos da mistura óleo-água visando a determinação da eficiência total de separação

\begin{tabular}{ccccc}
\hline Exp. & 4 & 5 & 6 & 7 \\
\hline $\mathrm{Q}_{\mathrm{t}}(\mathrm{L} / \mathrm{min})$ & 20,77 & 20,74 & 21,00 & 20,79 \\
$\mathrm{Q}_{\mathrm{o}}(\mathrm{L} / \mathrm{min})$ & 8,27 & 8,17 & 8,31 & 8,12 \\
$\mathrm{Q}_{\mathrm{a}}(\mathrm{L} / \mathrm{min})$ & 12,49 & 12,56 & 12,69 & 12,67 \\
$\mathrm{C}_{\mathrm{v} \text { (alim) }}$ & 0,39 & 0,39 & 0,39 & 0,39 \\
$\mathrm{C}_{\mathrm{v} \text { (ovf) }}$ & 0,87 & 0,92 & 0,91 & 0,86 \\
$\mathrm{C}_{\mathrm{v} \text { (und) }}$ & 0,03 & 0,09 & 0,20 & 0,27 \\
$\mathrm{R}_{\mathrm{FT}}$ & 0,43 & 0,36 & 0,26 & 0,19 \\
$\mathrm{R}_{\mathrm{F}}$ & 0,09 & 0,04 & 0,03 & 0,04 \\
$\mathrm{ET}$ & 0,95 & 0,84 & 0,62 & 0,42 \\
$\mathrm{ET}^{\prime}$ & 0,95 & 0,83 & 0,60 & 0,39 \\
\hline
\end{tabular}

Figura 7 - Eficiência total de separação versus a razão de fluido total

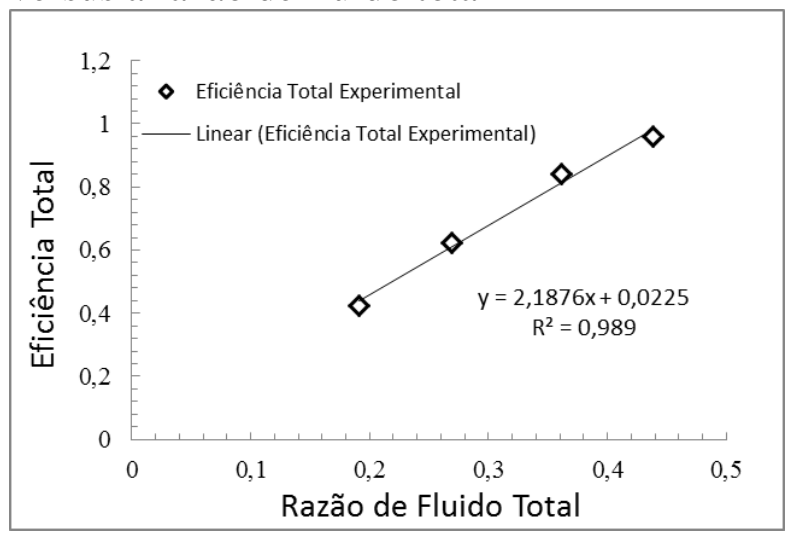

A Figura 7 mostra a existência de uma relação linear entre $\mathrm{R}_{\mathrm{FT}}$ e ET dada por:

$$
\mathrm{ET}=2,19 \mathrm{R}_{\mathrm{FT}}+0,02 \quad\left(\mathrm{R}^{2}=0,99\right)
$$

\section{CONCLUSÕES}

Um hidrociclone para a separação de misturas óleo-água contendo altos teores de óleo foi projetado por meio de simulações numéricas com fluidodinâmica computacional, construído e testado experimentalmente, gerando eficiências de separação de óleo de $93 \% \pm 2 \%$, para as condições experimentais apresentadas. Experimentos com frações volumétricas de $40 \%$ de óleo em água foram realizados em um 
sistema aberto com valores de vazão de entrada idênticos aos das simulações. Os experimentos apresentaram valores médios de eficiência de separação e razão de fluido com desvios abaixo de $2 \%$. A relação entre a razão de fluido total e a eficiência total de separação foi linear.

\section{NOMENCLATURA}

CFD - fluidodinâmica computacional (computational fluid dynamics) [-];

$\mathrm{C}_{\mathrm{V}}$ - concentração volumétrica (v/v) [-];

$\mathrm{C}_{\mathrm{V}}$ (alim) - concentração volumétrica na corrente de alimentação (v/v) [-];

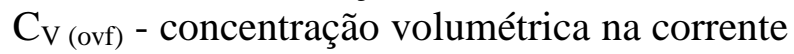
de overflow (v/v) [-];

$\mathrm{C}_{\mathrm{V}}$ (und) - concentração volumétrica na corrente de underflow (v/v) [-];

Dc - diâmetro da seção cilíndrica do hidrociclone [mm];

Di - diâmetro do duto de alimentação [mm];

Do - diâmetro do tubo de overflow [mm];

$\mathrm{Du}$ - diâmetro do underflow [mm];

ET - eficiência total do hidrociclone [-];

ET' - eficiência total reduzida do hidrociclone

$[-]$

$\mathrm{G}$ - eficiência granulométrica [-];

G' - eficiência granulométrica reduzida [-];

L1 - comprimento da parte cilíndrica do hidrociclone [mm];

$\mathrm{Q}_{\mathrm{o}}$ - vazão volumétrica de óleo na entrada do hidrociclone $\left[\mathrm{L} \mathrm{min}^{-1}\right.$;

$\mathrm{Q}_{\mathrm{a}}$ - vazão volumétrica de água na entrada do equipamento [ $\mathrm{L} \mathrm{min}^{-1}$;

$\mathrm{Q}_{\mathrm{t}}$ - vazão volumétrica total (óleo + água) [L $\left.\min ^{-1}\right]$;

$\mathrm{R}_{\mathrm{F}}$ - razão de fluido (vazão vol. de óleo no overflow / vazão vol. de óleo na entrada) [-];

$\mathrm{R}_{\mathrm{FT}}$ - razão de fluido total (vazão vol. total do overflow / vazão vol. total na entrada) [-];

VF - comprimento do vortex-finder do hidrociclone [mm];

$\rho$ - densidade do fluido $\left[\mathrm{kg} \mathrm{m}^{-3}\right]$;

$\Theta_{2}$ - ângulo da segunda seção cônica do hidrociclone [rad]; $\mu$ - viscosidade dinâmica do fluido $\left[\mathrm{kg} \mathrm{m}^{-1} \mathrm{~s}^{-1}\right]$;

\section{REFERÊNCIAS}

ALMEIDA, L.C.; OLIVEIRA JR., J.A.A.; MEDRONHO, R.A., Simulação numérica da separação água-óleo em hidrociclones para baixas frações de óleo. Anais do PDPETRO 2009 - $5^{\circ}$ Congresso Brasileiro de Pesquisa e Desenvolvimento em Petróleo e Gás, Fortaleza, 2009.

ALVES, J. V. B., MEDRONHO, R. A., Hidrociclone para a separação do óleo residual de água em refinarias. Anais do COBEQ 2012 - XIX Congresso Brasileiro de Engenharia Química, ABEQ, Búzios, p. 10958 - 10967, 2012.

ANJOS, R. P., OLIVEIRA, R. S., ALVES, J. V. B., MEDRONHO, R. A., Influência do número de entradas tangenciais e do ângulo da seção cônica de um hidrociclone no tratamento de águas oleosas. Anais do COBEQ 2012 - XIX Congresso Brasileiro de Engenharia Química, ABEQ, Búzios, 2012.

ARAÚJO, C. A. O., OLIVEIRA, R. S., SCHEID, C. M., MEDRONHO, R. A., Hidrociclones para a separação de dispersões óleo-água com altos teores de óleo: um estudo com CFD. Anais do ENEMP 2013 - XXXVI Congresso Brasileiro de Sistemas Particulados, Maceió, 2013.

ARAÚJO, C. A. O., BARCELlOS, J. P. M., SCHEID, C. M., MEDRONHO, R. A., Efeito de variáveis geométricas no desempenho de hidrociclones para a separação de águas oleosas com alto teor de óleo. Anais do COBEQ 2012 - XIX Congresso Brasileiro de Engenharia Química, ABEQ - Associação Brasileira de Engenharia Química, Búzios, p.5896 - 5904, 2012. 
BAI, ZHI-SHAN; WANG, HUA-LIN; TU, SHAN-TUNG; Oil-water separation using hydrocyclones enhanced by air bubbles, Chemical Engineering Research and Design, 89, 55-59, 2011.

BRAGA, E. R., CAMPOS, J. B. L. M., SCHEID, C. M., MEDRONHO, R. A., Perfil de velocidades tangenciais em um hidrociclone para separação líquido/líquido. Anais do ENEMP 2013 - XXXVI Congresso Brasileiro de Sistemas Particulados, Maceió, 2013.

COELHO, D. B., ALVES, J. V. B., MEDRONHO, R. A., Desempenho de um Hidrociclone para a Separação de Águas Oleosas. Anais do PDPetro 2011 - $6^{\circ}$ Congresso Brasileiro de Pesquisa e Desenvolvimento em Petróleo e Gás, ABPG - Associação Brasileira de P\&D em Petróleo e Gás, Florianópolis, 2011.

COELHO, D. B., MEDRONHO, R. A., ALVES, J. V. B., A eficiência de separação de um hidrociclone para águas oleosas pelas abordagens lagrangeana e euleriana. Anais do COBEQ 2012 - XIX Congresso Brasileiro de Engenharia Química, $\mathrm{ABEQ}$, Búzios, p.7411 - 7420, 2012.

FREITAS, A. G. B.; Modelagem e simulação do tratamento de água oleosa usando hidrociclone, Dissertação (Mestrado em Engenharia Química) - Universidade Federal de Sergipe, São Cristóvão, 2009.

HASHMI, K. A., HAMZA, H. A., WILSON, J. C., Canmet hydrocyclone: an emerging alternative for the treatment of oily waste streams, Minerals Engineering, 643649, 2004.

MENDONÇA, A. C. L. T., ARAÚJO, C. A. O., MEDRONHO, R. A., O hidrociclone na separação óleo/água: um estudo com o auxílio de fluidodinâmica computacional. Anais do PDPetro 2013 - $7^{\circ}$ Congresso Brasileiro de Pesquisa e Desenvolvimento em Petróleo e Gás, ABPG - Associação Brasileira de P\&D em Petróleo e Gás, Aracaju, 2013.

MORAES, C. A. C., Curso de Hidrociclones e Sistemas de Separação Ciclônica. Rio e Janeiro: Cenpes. Universidade Coorporativa. Agosto 2006.

NEZHATI, K.; THEW, M.T.; Aspects of the performance and scaling of hydrocyclones for use with light dispersions, $3^{\text {rd }}$ International Conference on Hydrocyclones, 167-180, September, 1987.

QUEIROZ, F. V., MEDRONHO, R. A., Simulação numérica de hidrociclones para a separação de misturas de alto teor de água em óleo como fase contínua. Anais do COBEQ 2012 - XIX Congresso Brasileiro de Engenharia Química, ABEQ - Associação Brasileira de Engenharia Química, Búzios, p.5557 - 5566, 2012.

ZHOU, N.; GAO, Y.; AN, W.; YANG, M.; Investigation of velocity Field and oil distribution in an oil-water hydrocyclone usin a particle dynamics analyzer, Chem. Eng. J., n. 157 , p. 73-79, 2010.

\section{AGRADECIMENTOS}

Os autores agradecem ao Laboratório de Fluidodinâmica Computacional (LabCFD), ao Núcleo Interdisciplinar da Dinâmica de Fluidos (NIDF), ambos situados no campus da UFRJ, e agradecem também ao Laboratório de Escoamento de Fluidos Giulio Massarani (LEF), no campus da UFRRJ, pela extensa colaboração neste trabalho. 